\title{
Highly Rectifying Fluidic Diodes Based on Asymmetric Layer-by- Layer Nanofilms on Nanochannel Membranes
}

Shouwei Zhang ${ }^{\mathrm{a}}$, Liu Yang ${ }^{\mathrm{b}}$, Dong ding ${ }^{\mathrm{c}}$, Pengcheng Gao ${ }^{\mathrm{a}}$, Fan Xia*a, Merlin L. Bruening ${ }^{\mathrm{b}, \mathrm{c}}$

${ }^{a}$ Faculty of Materials Science and Chemistry, China University of Geosciences, 430074 Wuhan, China

${ }^{\mathrm{b}}$ Department of Chemistry and Biochemistry, University of Notre Dame, Notre Dame, Indiana 46556, United States

${ }^{\mathrm{c}}$ Department of Chemical and Biomolecular Engineering, University of Notre Dame, Notre Dame, Indiana 46556, United States

*Email: xiafan@cug.edu.cn; xiafan@hust.edu.cn 


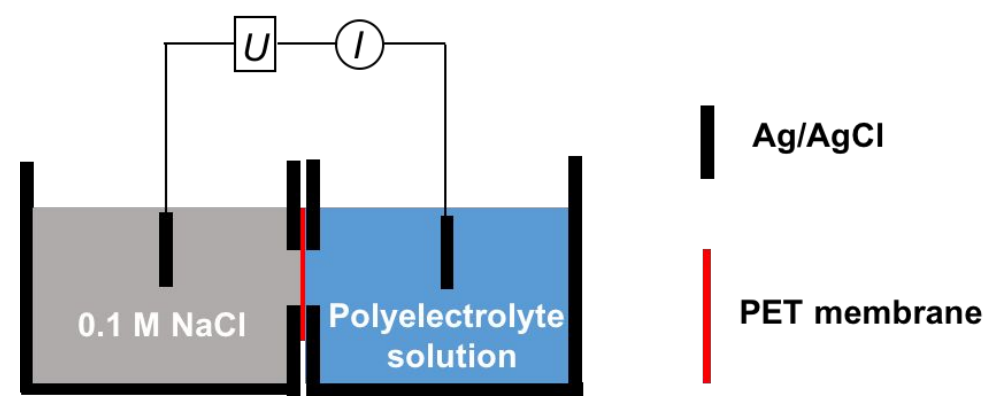

Figure S1. Illustration of the electrochemical measurement configuration. For in-situ monitoring of film formation, the left cell was filled with $0.1 \mathrm{M} \mathrm{NaCl}(\mathrm{pH} 8)$, and the right cell contained polyelectrolyte in $0.1 \mathrm{M} \mathrm{NaCl}(\mathrm{pH} \mathrm{8).} \mathrm{After} \mathrm{adding} \mathrm{the}$ polyelectrolyte solution, I-V curves were collected at 5-min intervals for $15 \mathrm{~min}$. Afterward, fresh $0.1 \mathrm{M} \mathrm{NaCl}$ solution was employed to rinse the right cell two times (with a 2 min exposure for each rinse) followed by measurement of I-V curves in fresh $0.1 \mathrm{M} \mathrm{NaCl}$ before subsequent deposition of oppositely charged polyelectrolyte. The grounded electrode was placed in the $0.1 \mathrm{M} \mathrm{NaCl}$ solution side (left cell).

\begin{tabular}{|c|} 
(a) $\quad$ Model \\
\hline Ambient: air \\
\hline Substrate: B-spline $(\mathrm{Au})$ \\
\hline
\end{tabular}

(b)

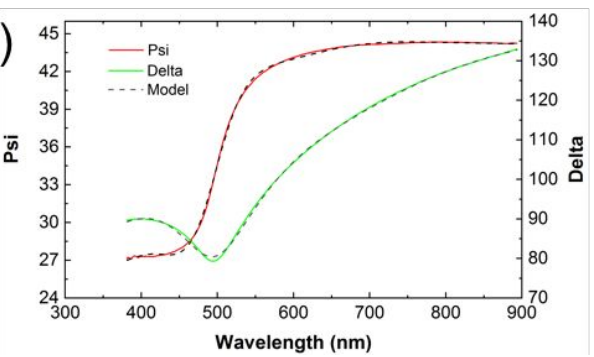

(C)

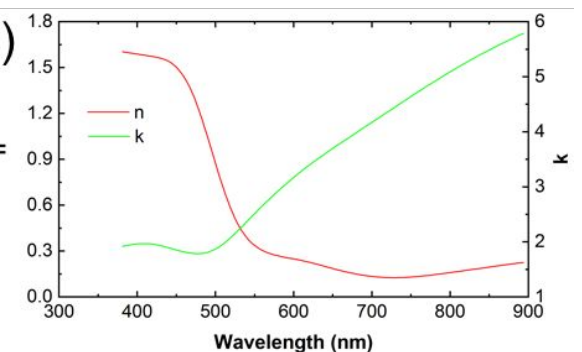

Figure S2. Example data for determining the optical constants of a gold substrate. (a) Ellipsometric model, (b) Plot of fitted (dashed lines) and measured values of the ellipsometric parameters delta (green) and psi (red), and (c) The resulting optical constants for the gold layer (substrate) in air.

The optical constants of gold films vary slightly with the technique employed to deposit them. Nevertheless, accurate optical constants $(n, k)$ for the Au substrate are vital in model fitting to characterize films on these substrates. Using a B-spline dielectric 
function, we fitted the ellipsometric data from reflection from a gold surface to obtain accurate values of $\mathrm{n}$ and $\mathrm{k}$.

(a)

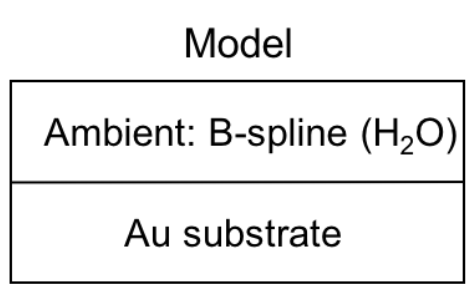

(b)

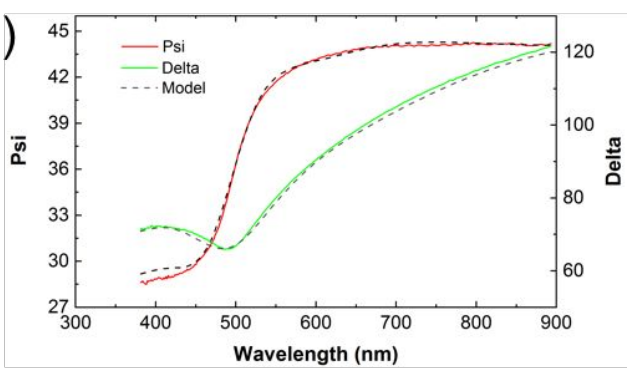

(d)

(c)

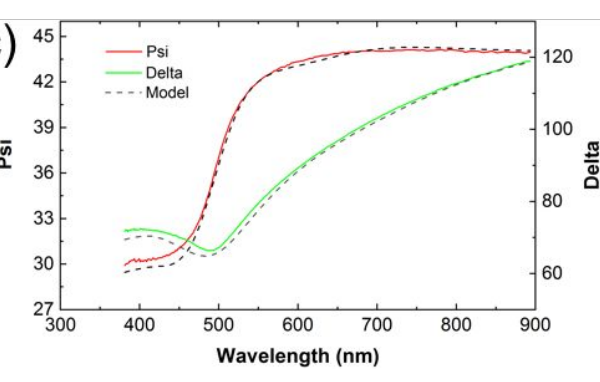

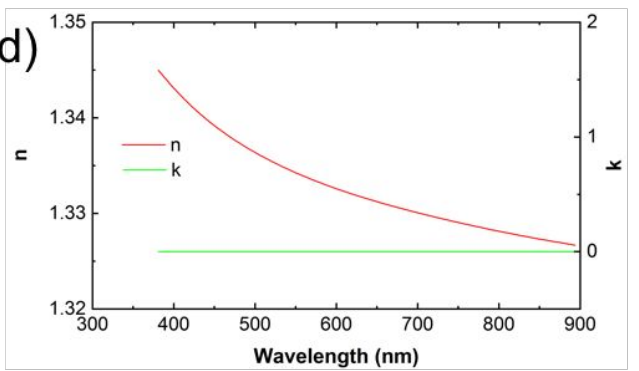

Figure S3. Example ellipsometry data for determining the optical constants of water during reflection from a Au-coated substrate. (a) Model; (b, c) Plot of fitted (dashed lines) and measured values of the ellipsometric parameters delta (green) and psi (red) in water at $\mathrm{pH} 3$ (b) and $\mathrm{pH} 8$ (c); (d) Optical constants for water (ambient) at $\mathrm{pH} 3$ and $\mathrm{pH}$ 8. Built-in optical constants of water served as the starting material in the B-spline dielectric function. The reflective index of water (d) is essentially the same, regardless of the $\mathrm{pH}$ value, while $k$ is set to be zero. 
(b)

(a)

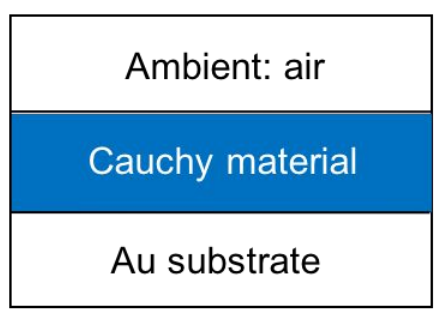

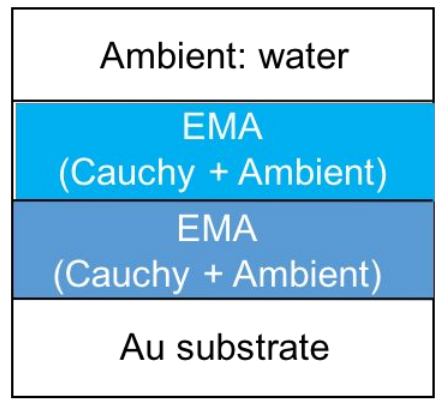

(c)

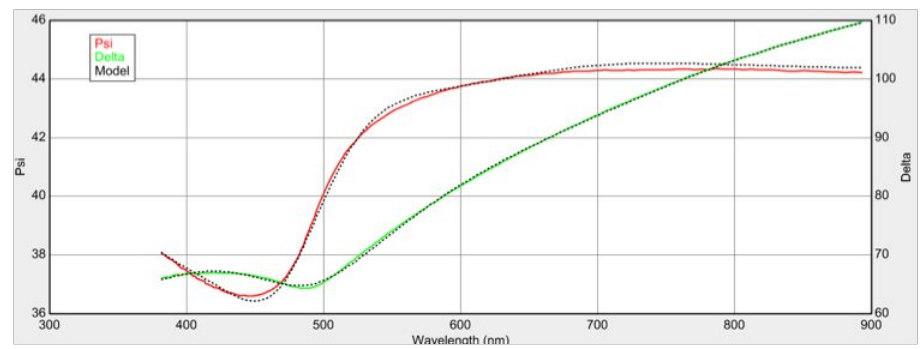

(d)

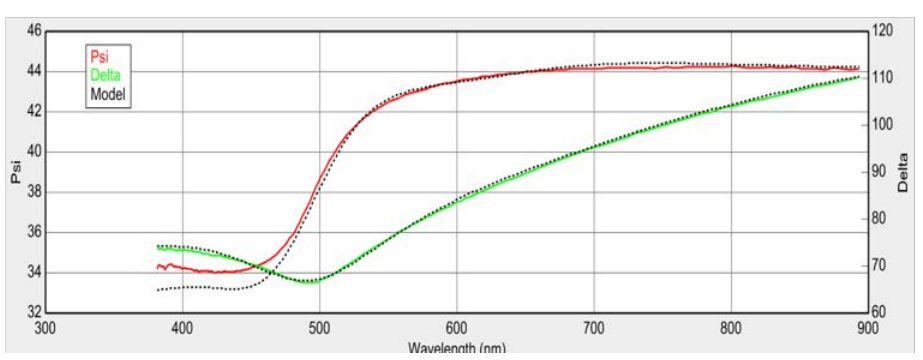

Figure S4. Example ellipsometry data for determining the thickness and volume fraction of water in LbL films. Ellipsometry models for LbL films in (a) air and (b) in water. The number of effective medium approximation (EMA) layers depends on the film structure as well as the agreement of experimental- and model-generated $\psi$ and $\Delta$ values. (c, d) Plots of fitted (dashed lines) and measured values of the ellipsometric parameters delta (green) and psi (red) for $(\mathrm{PEI} / \mathrm{CS})_{4} /$ Crosslinking/(PEI/CS) $3 / \mathrm{PEI}$ film in air (c) and in water $\mathrm{pH} 8$ (d).

The Cauchy material (polyelectrolyte) uses equation S1 to model the refractive index, $n(\lambda)$

$n(\lambda)=A+\frac{B}{\lambda^{2}}$

Equation S1

where $\lambda$ is the wavelength of incident light (nm), and for the polyelectrolyte materials, A (dimensionless) and B ( $\left.\mathrm{nm}^{2}\right)$ are fitted values starting with initial guesses of 1.5 and 0.01 , respectively. The value of $k$ is 0 . 
(a)<smiles>CCN(CCN)CCNCCN(CCNCCN)CCN(CCNCCN)CCN(CCN)CCN</smiles>

(b)

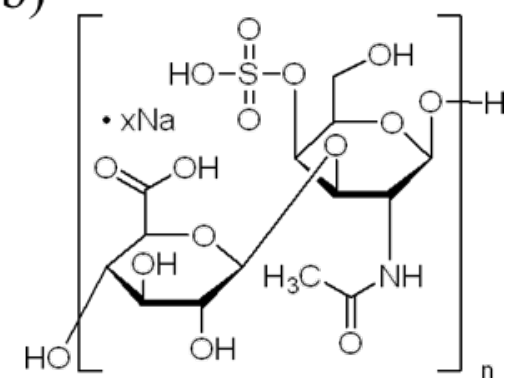

Figure S5. Structures of (a) neutral branched poly(ethyleneimine) (PEI) and (b) fully protonated chondroitin-4-sulfate (CS).

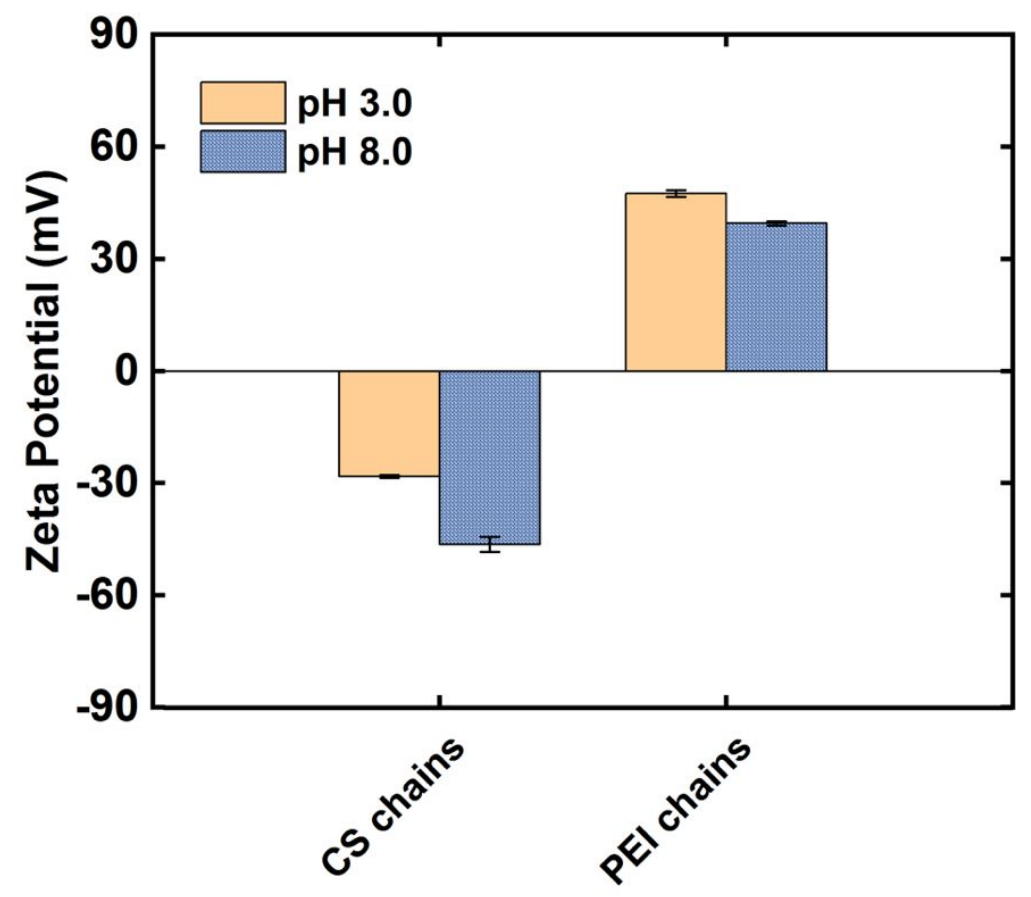

Figure S6. Zeta potentials of CS and PEI chains in water at $\mathrm{pH} 3$ and 8. 

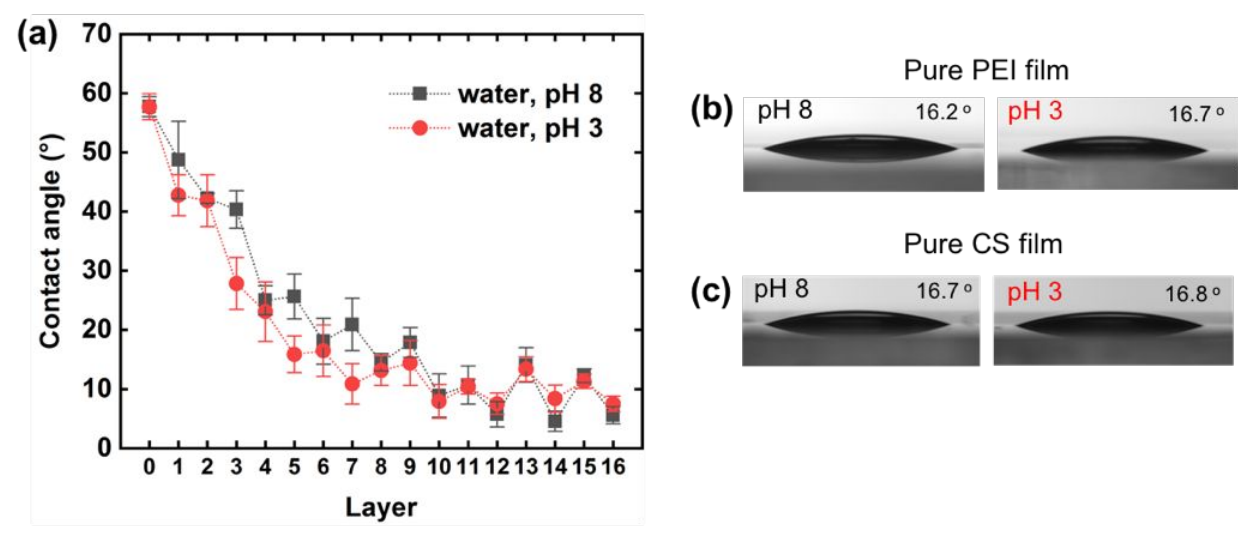

(d)

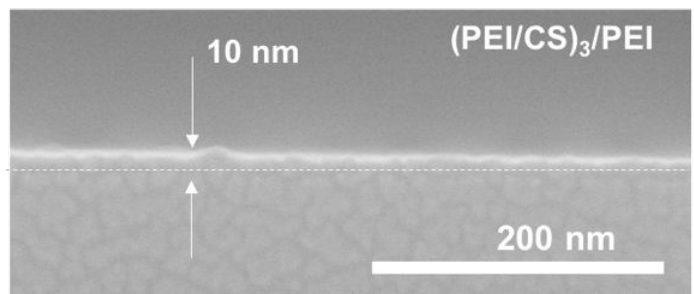

Figure S7. (a) In-situ monitoring of the contact angle during the formation of (PEI/CS) $)_{4} /$ Crosslinking/(PEI/CS) 3 /PEI films on nanochannel membranes. (b) Contact angles of pure PEI and CS films. These pure films were prepared by placing drops of polyelectrolyte solutions on PET membranes followed by drying in an $80{ }^{\circ} \mathrm{C}$ oven overnight. (c) Cross-sectional SEM image of a (PEI/CS) 3 /PEI film, showing a uniform coating. The dash line indicates the interface between silicon and the polyelectrolyte film. 
(a)

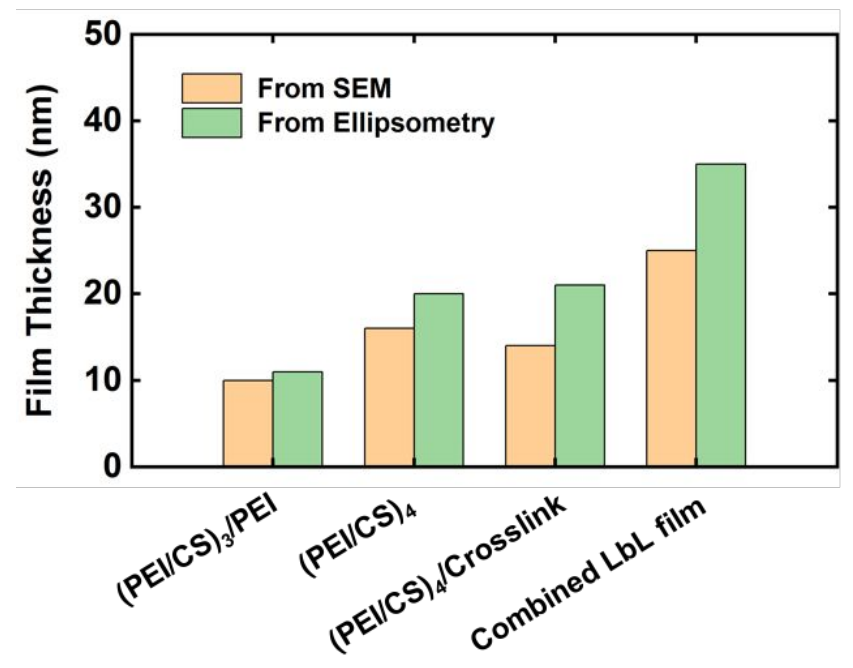

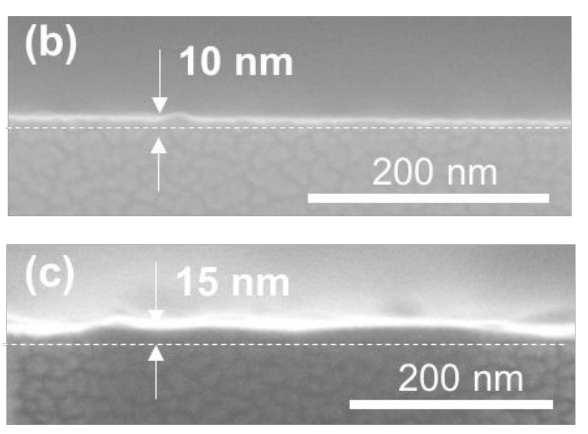
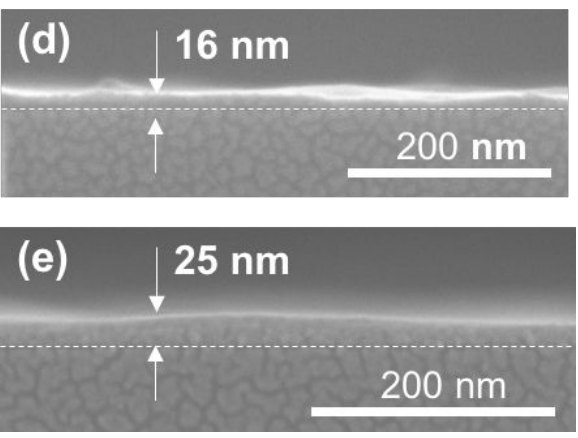

Figure S8. (a) Ellipsometric and SEM thicknesses of LbL films fabricated on silicon wafers. For ellipsometry, Au-coated silicon wafers were employed as substrates. (b-f) Cross-sectional SEM images of films on silicon wafers were obtained to calculate film thickness. (b) $\quad(\mathrm{PEI} / \mathrm{CS})_{3} / \mathrm{PEI}, \quad$ (c) $\quad(\mathrm{PEI} / \mathrm{CS})_{4}, \quad$ (d) $\quad(\mathrm{PEI} / \mathrm{CS})_{4} /$ Crosslinking, (e) (PEI/CS) $)_{4} /$ Crosslinking/(PEI/CS) $3 /$ PEI (combined film).

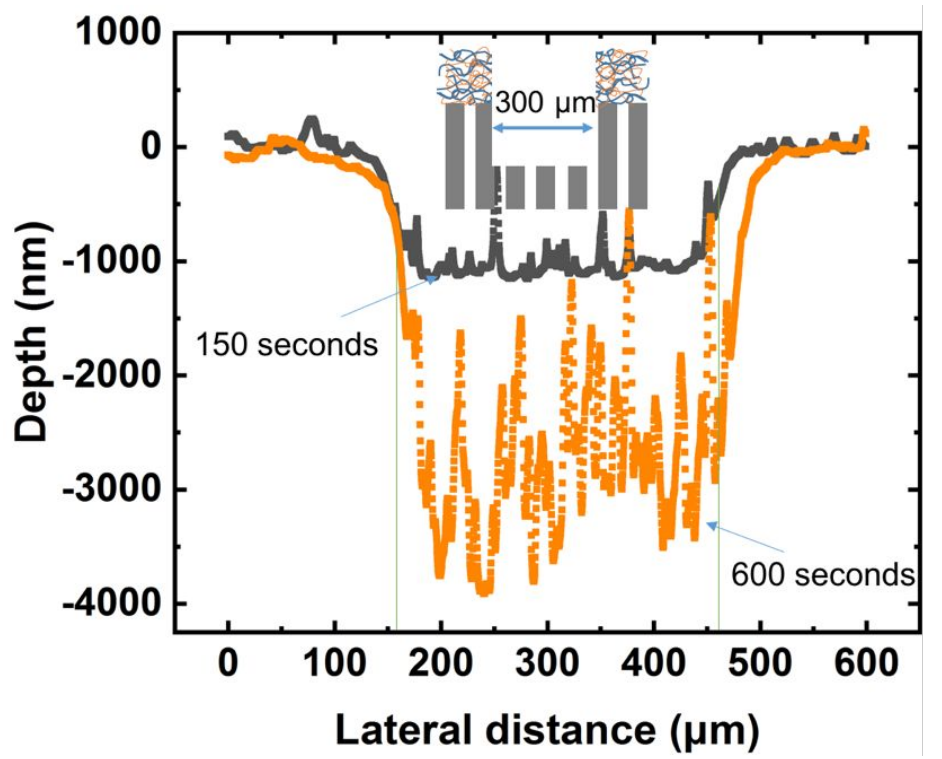

Figure S9. TOF-SIMS depth profiles of the etched area on a $(\mathrm{PEI} / \mathrm{CS})_{4} /$ Crosslinking/(PEI/CS $)_{3} / \mathrm{PEI}$ film over a PET membrane. 


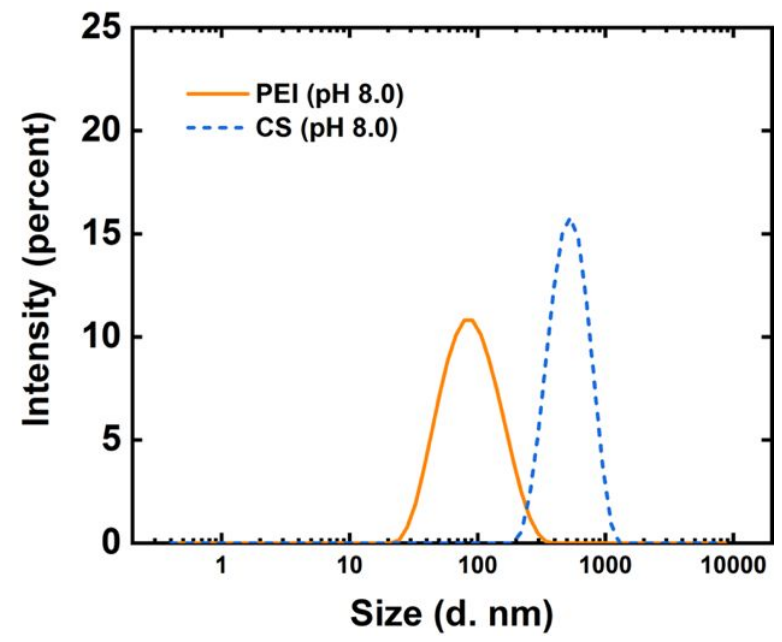

Figure S10. Dynamic light scattering (DLS) measurements of hydrodynamic diameters of PEI and CS polyelectrolyte chains in a $0.1 \mathrm{M} \mathrm{NaCl}$ solution ( $\mathrm{pH} 8)$.

(a)

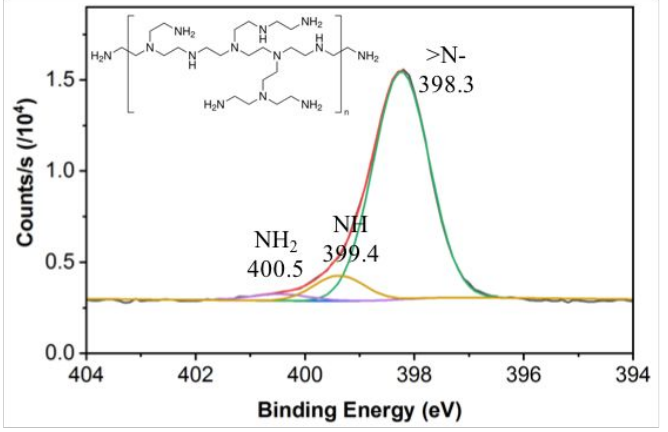

(b)

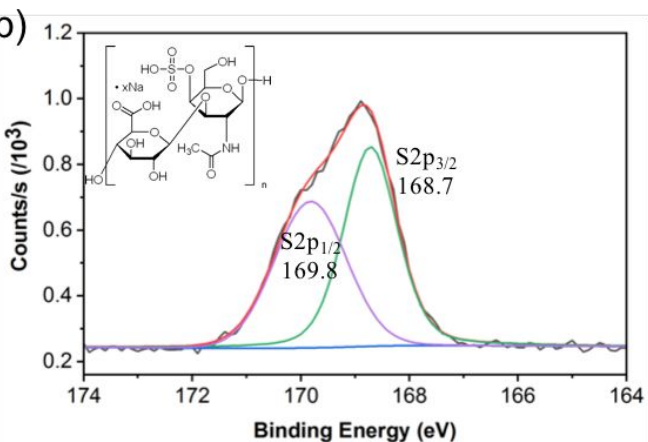

(c)

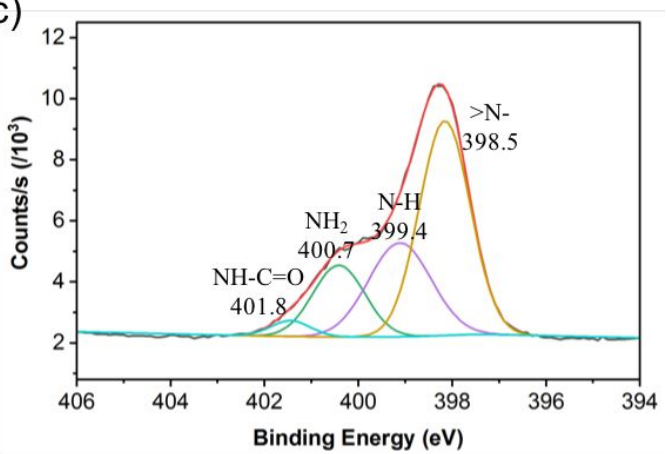

(d)

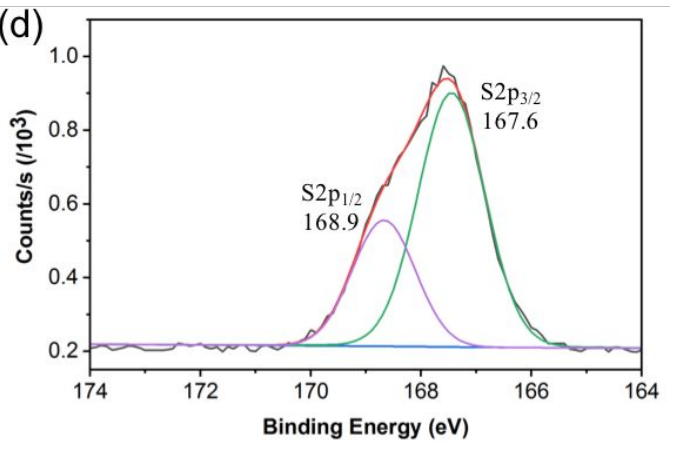

Figure S11. X-ray photoelectron (XPS) narrow scan $\mathrm{N}_{1 \mathrm{~s}}(\mathrm{a}, \mathrm{c})$ and $\mathrm{S}_{2 \mathrm{p}}(\mathrm{b}, \mathrm{d})$ spectra for (a) PEI, (b) $\mathrm{CS}$ and (c,d) (PEI/CS) ${ }_{4} /$ Crosslinking/(PEI/CS) $3 / \mathrm{PEI}$ films, respectively. The black lines indicate the experimental data, while the colored lines denote the fitted curves. 


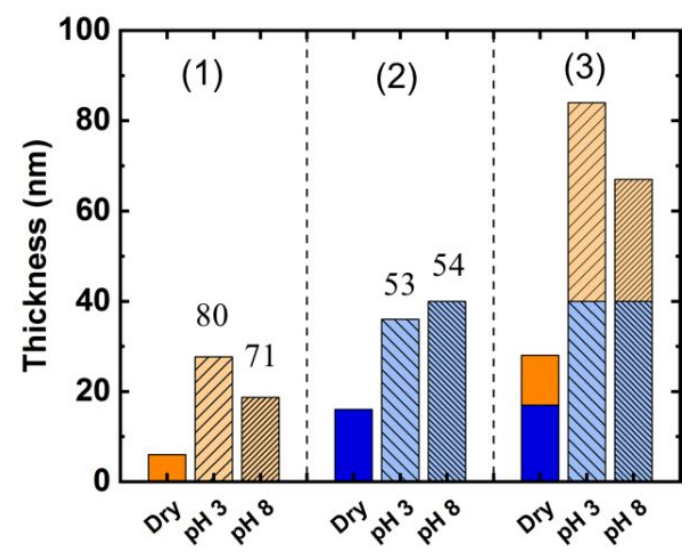

Figure S12. Ellipsometric dry and hydrated thicknesses of LbL films. (1) (PEI/CS) $3 / \mathrm{PEI}$; (2) Crosslinked $(\mathrm{PEI} / \mathrm{CS})_{4}$; (3) $(\mathrm{PEI} / \mathrm{CS})_{3} / \mathrm{PEI} /$ crosslinking/(PEI/CS $)_{3} / \mathrm{PEI}$. Numeric values shown above some of the columns are water fractions in those films. The different regions for film (3) represent the thicknesses of the two different EMA layers. For these films, we could not fit the data using a single EMA layer. The refractive indices for the two different regions in water ( $\mathrm{pH} 8)$ are $\sim 1.42$ and $\sim 1.37(623 \mathrm{~nm})$ for the crosslinked and non-crosslinked regions, respectively.

(a)

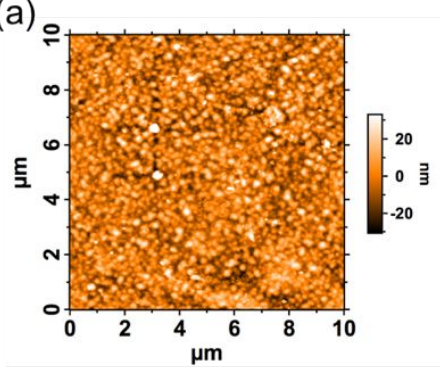

(b)

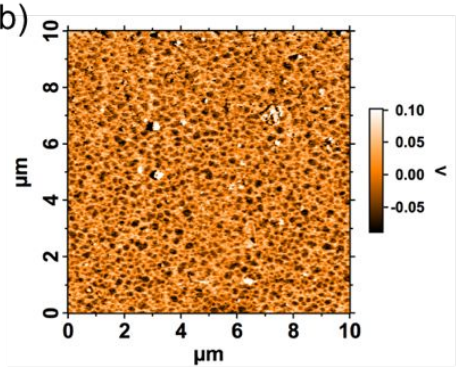

(c)

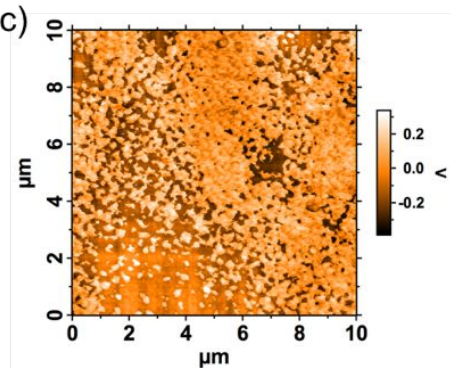

Figure S13. (a) AFM topography and $(\mathrm{b}, \mathrm{c})$ moduli of $(\mathrm{PEI} / \mathrm{CS})_{4} /$ Crosslinking $/(\mathrm{PEI} / \mathrm{CS})_{3} / \mathrm{PEI}$ films on membranes in $(\mathrm{a}, \mathrm{b}) \mathrm{pH} 3$ and (c) $\mathrm{pH} 8$ solutions. 

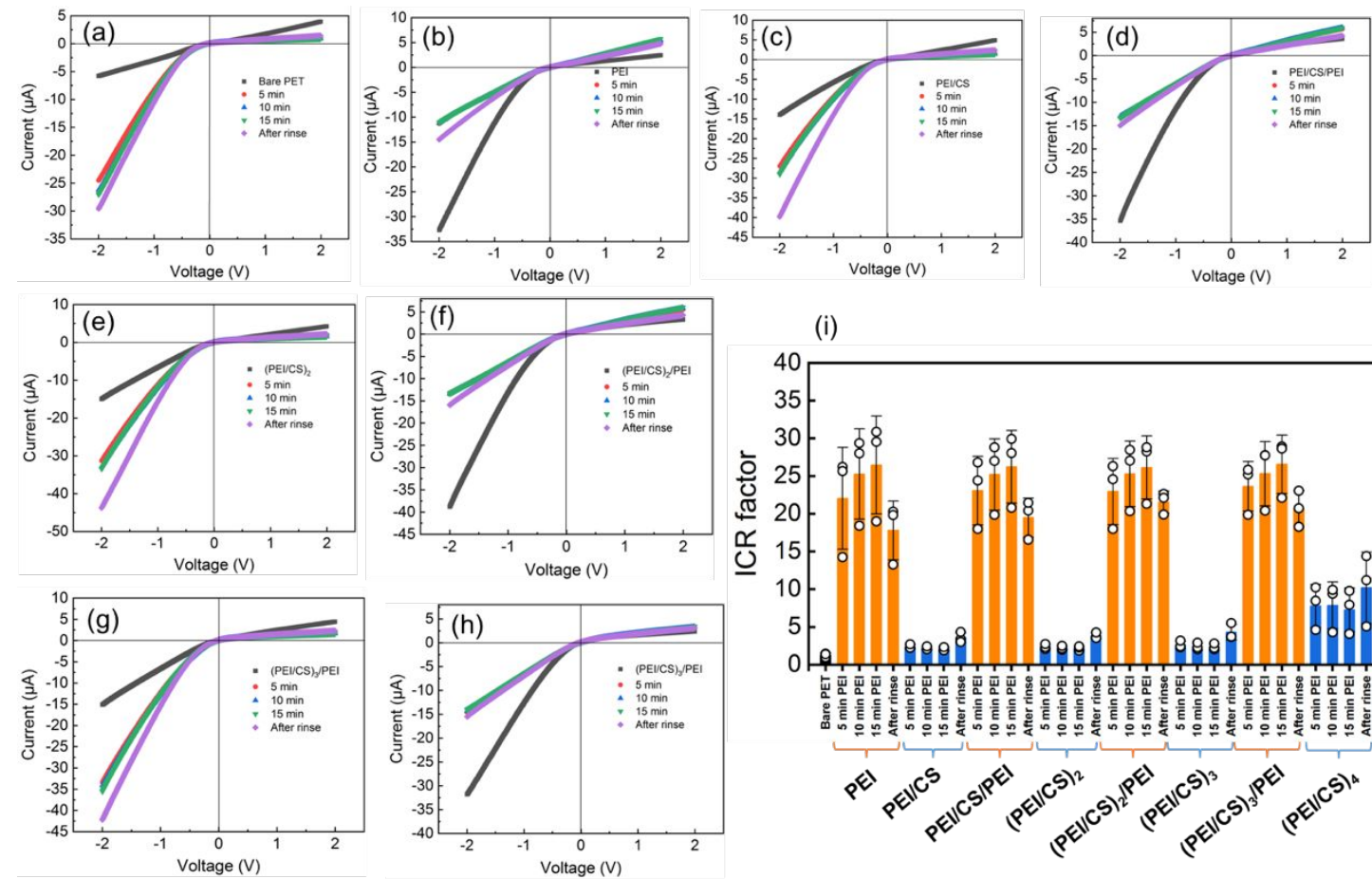

(i)

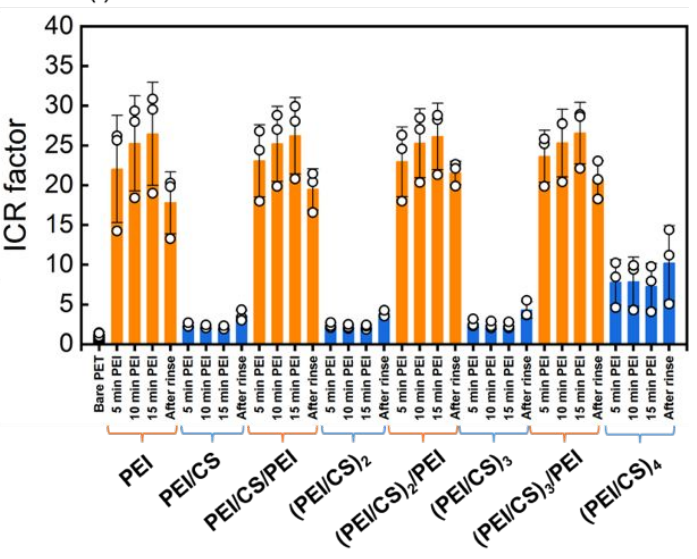

Figure S14. In situ monitoring of LbL adsorption using I-V curves (from $-2 \mathrm{~V}$ to $+2 \mathrm{~V}$ ) collected over 5 min intervals during formation of a (PEI/CS $)_{4}$ film on a PET membrane with $10 \mathrm{~nm}$ pores. The figures show data for adsorption of (a) PEI directly on the membrane, (b) CS on the first PEI layer, (c) PEI on PEI/CS, (d) CS on PEI/CS/PEI, (e) $\mathrm{PEI}$ on $(\mathrm{PEI} / \mathrm{CS})_{2}$, (f) $\mathrm{CS}$ on $(\mathrm{PEI} / \mathrm{CS})_{2} / \mathrm{PEI},(\mathrm{g}) \mathrm{PEI}$ on $(\mathrm{PEI} / \mathrm{CS})_{3}$, and (h) CS on $(\mathrm{PEI} / \mathrm{CS})_{3} / \mathrm{PEI}$. The black line in each figure is the membrane prior to adsorption of the layer of interest. Figure (i) summarizes the ion-current rectification factors as a function of adsorption time for each layer. Adsorption employed $1 \mathrm{mg} / \mathrm{ml}$ polyelectrolyte solutions with $0.1 \mathrm{M} \mathrm{NaCl}(\mathrm{pH} 8)$. Uncertainties in Figure (i) are the standard deviations of values for three independent membranes, and each circle represents an independent membrane. 

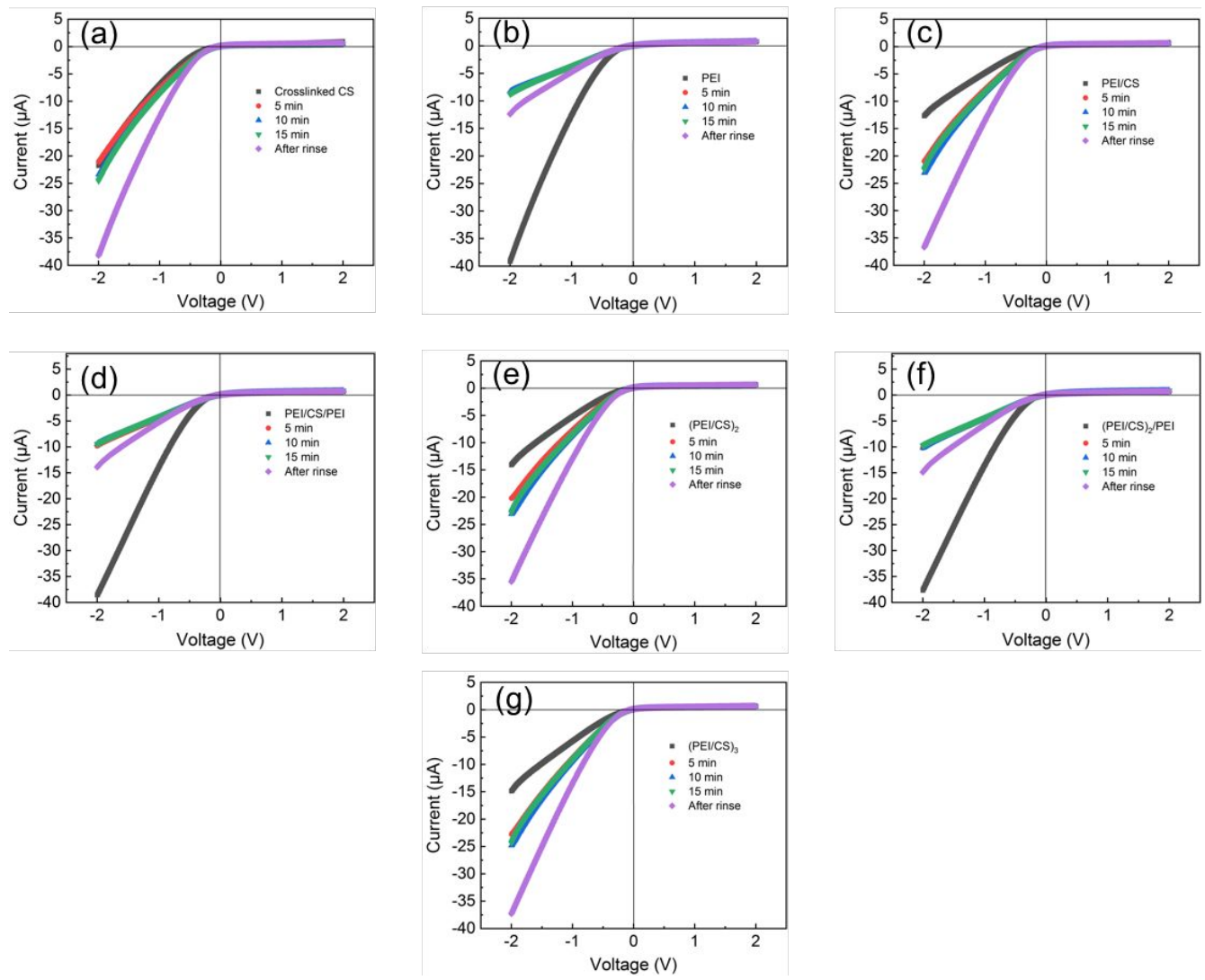

Figure S15. In situ monitoring of LbL adsorption on crosslinked (PEI/CS) $)_{4}$ films on PET membranes. Monitoring employed I-V curves $(-2 \mathrm{~V}$ to $+2 \mathrm{~V})$ collected at $5 \mathrm{~min}$ intervals. The figures show data for adsorption of (a) PEI on crosslinked (PEI/CS $)_{4}$, (b) $\mathrm{CS}$ on (PEI/CS) $)_{4} /$ Crosslinking/PEI, (c) PEI on (PEI/CS) ${ }_{4} /$ Crosslinking $/ \mathrm{PEI} / \mathrm{CS}$, (d) CS on (PEI/CS) $)_{4} /$ Crosslinking/PEI/CS/PEI, (e) PEI on (PEI/CS) $)_{4} /$ Crosslinking/(PEI/CS $)_{2}$, (f) $\mathrm{CS}$ on $(\mathrm{PEI} / \mathrm{CS})_{4} /$ Crosslinking/(PEI/CS $)_{2} / \mathrm{PEI}, \quad(\mathrm{g}) \quad \mathrm{PEI}$ on $(\mathrm{PEI} / \mathrm{CS})_{4} /$ Crosslinking/(PEI/CS $)_{3}, \quad$ and (h) $\mathrm{CS}$ on (PEI/CS $)_{4} /$ Crosslinking/(PEI/CS) 3 /PEI. Adsorption employed $1 \mathrm{mg} / \mathrm{ml}$ polyelectrolyte solutions $(\mathrm{pH} 8)$ in $0.1 \mathrm{M} \mathrm{NaCl}$. These are examples of measurements on single membrane. Experiments with three independent membranes were used to give the average values in Figure 2d. 


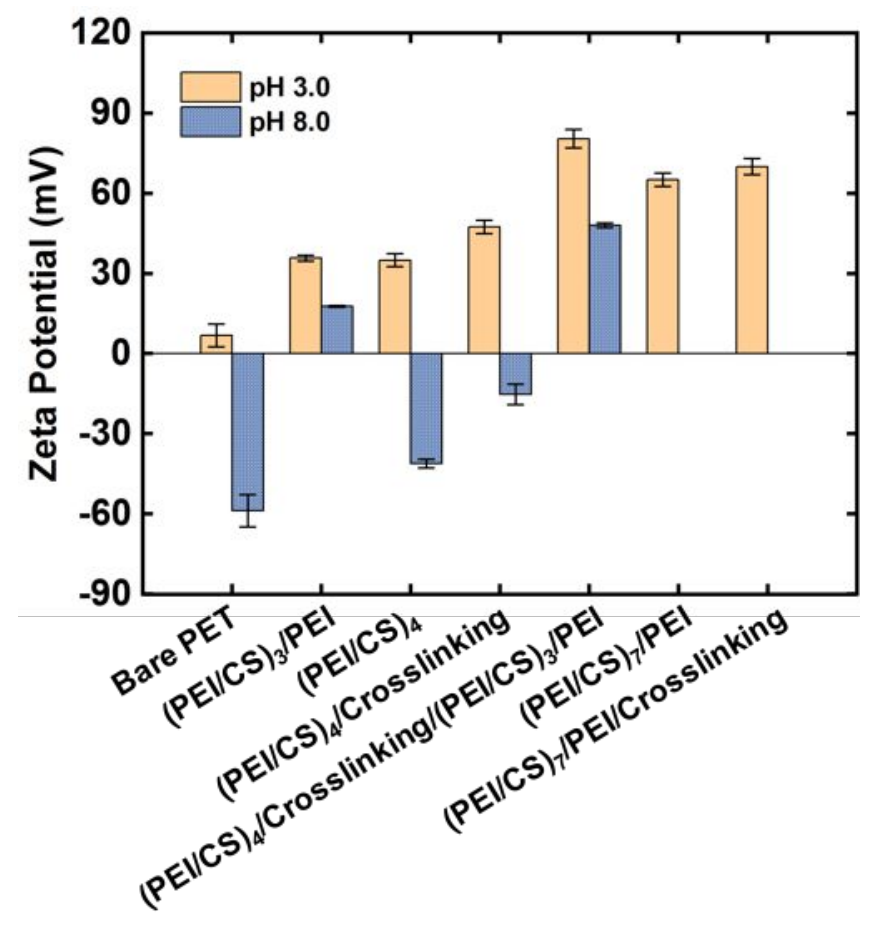

Figure S16. Zeta potentials of bare and film-modified membranes in $1 \mathrm{mM} \mathrm{KCl}$ at $\mathrm{pH}$ 3 and 8.

(a)

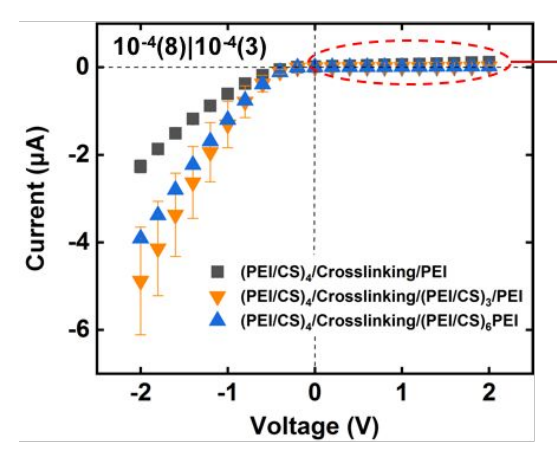

(b)

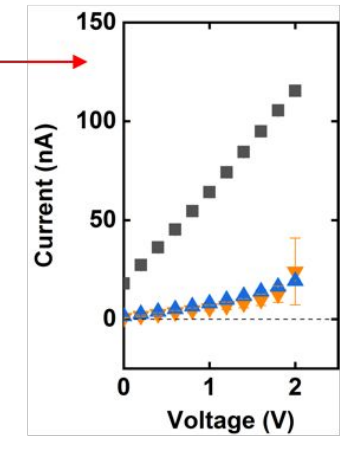

(c)

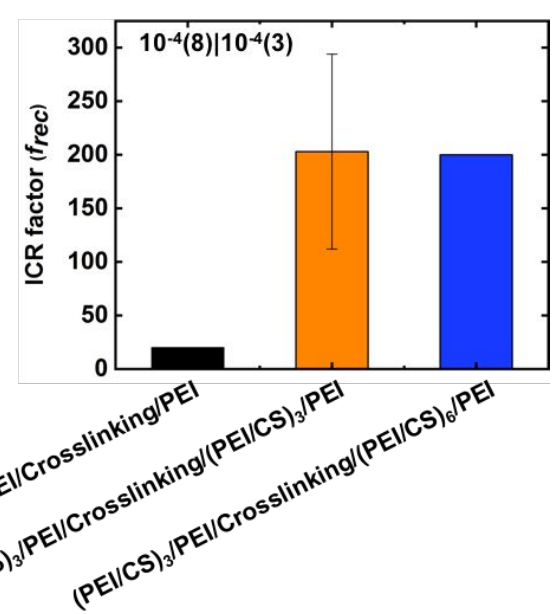

Figure S17. (a) I-V curves of different membranes collected in the $10^{-4}(8) \mid 10^{-4}$ (3) condition, (b) enlarged scale showing currents at positive voltages, (c) ICR factors obtained from figure (a). The control experiments with (PEI/CS $)_{4} / \mathrm{Crosslinking} / \mathrm{PEI}$ and (PEI/CS $)_{4} /$ Crosslinking/(PEI/CS $)_{6} / \mathrm{PEI}$ films were performed only once. 


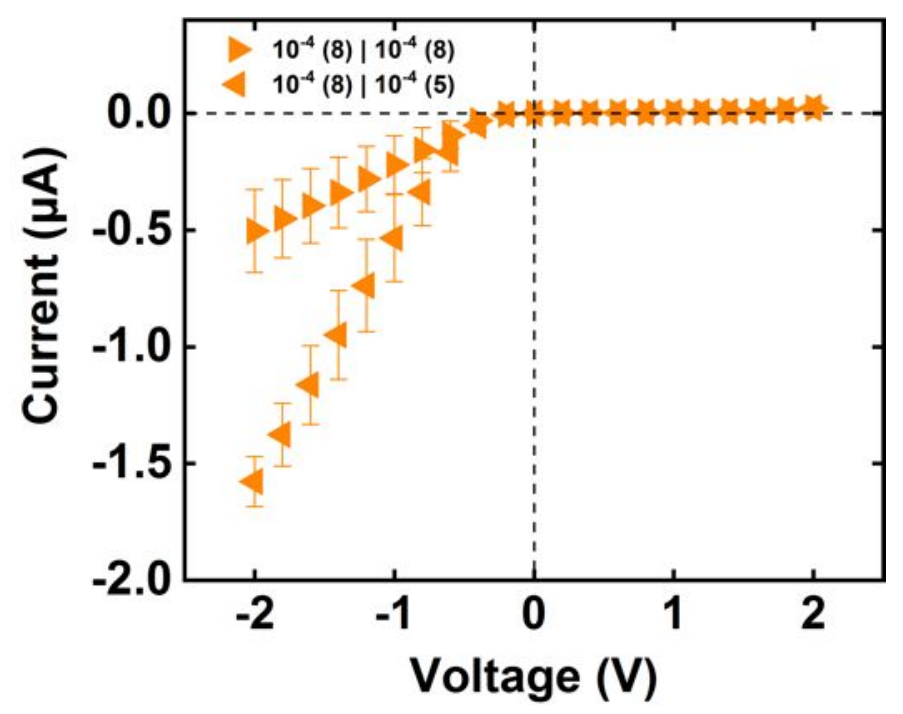

Figure S18. I-V curves for $\left.\quad(\mathrm{PEI} / \mathrm{CS})_{4} / \mathrm{Crosslinking/(PEI/CS}\right)_{3} / \mathrm{PEI}$-modified membranes using two different $\mathrm{pH}$ values $(5$ or 8$)$ on the film side of the membrane.

(a)

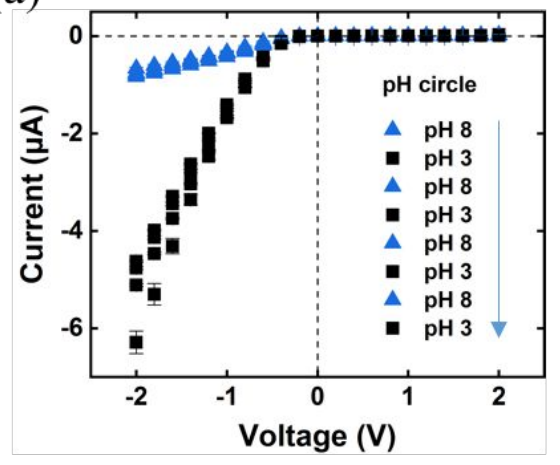

(b)

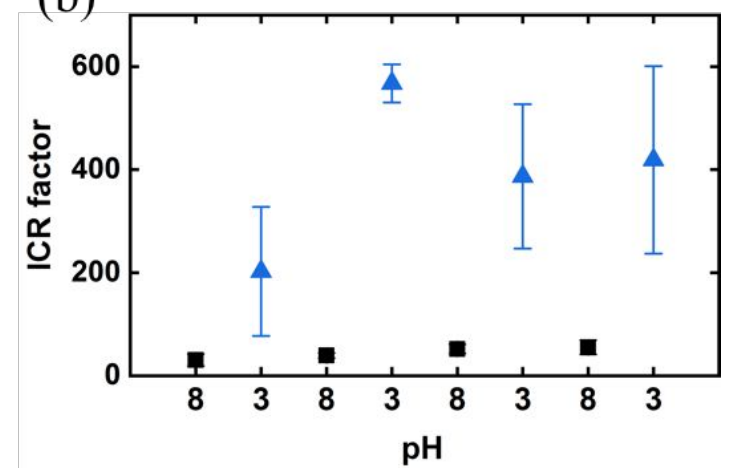

Figure S19. I-V curves collected in $10^{-4} \mathrm{M} \mathrm{NaCl}$ solution. The $\mathrm{pH}$ at the PET side was 8 , while the $\mathrm{pH}$ at the LbL film side alternated between 3 and 8 for several cycles. Data were obtained with a $(\mathrm{PEI} / \mathrm{CS})_{4} / \mathrm{Crosslinking} /(\mathrm{PEI} / \mathrm{CS})_{3} / \mathrm{PEI}$-modified PET membrane. The uncertainties in (b) come from only one sample with several scans. The ICR factor is very sensitive to the current at $2 \mathrm{~V}$. Current variations of only several tens of $\mathrm{nA}$ at $2 \mathrm{~V}$ will result in a large variation in the ICR factor. 

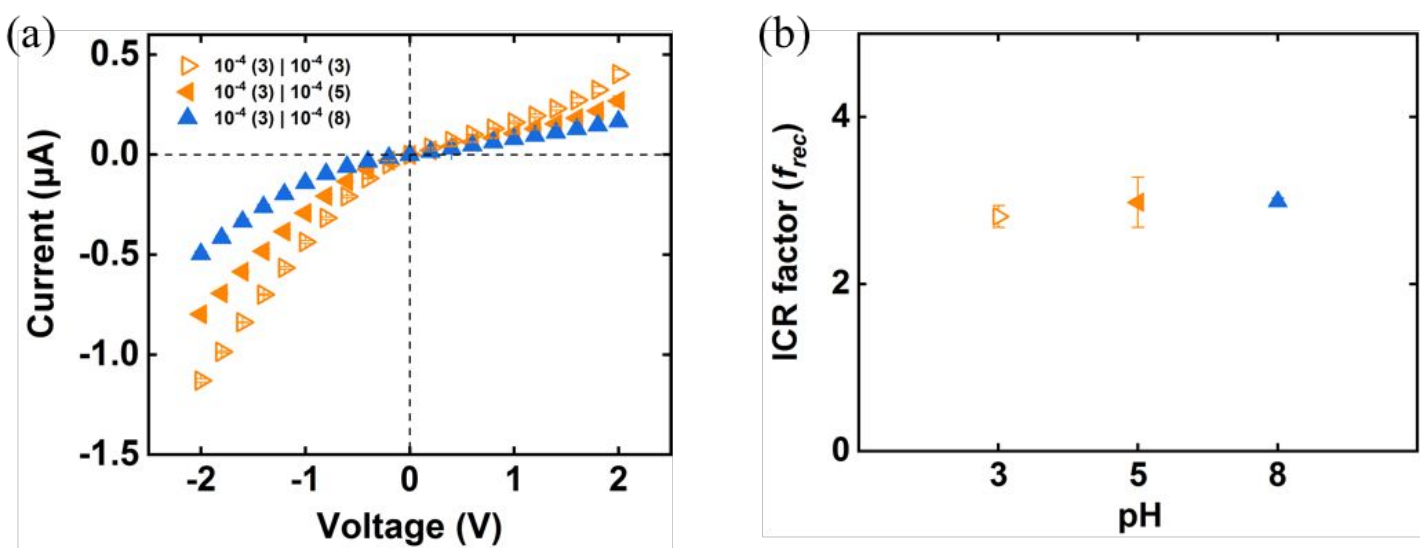

Figure S20. (a) I-V curves and (b) ICR factors for $(\mathrm{PEI} / \mathrm{CS})_{4} /$ Crosslinking/(PEI/CS $)_{3} /$ PEI-modified PET membranes in $10^{-4} \mathrm{M} \mathrm{NaCl}$ solutions with different $\mathrm{pH}$ combinations on the two sides of the membrane. The $\mathrm{pH}$ at the PET side of the membrane was 3, while the LBL film side has $\mathrm{pH}$ values of 3, 5 and 8. 